\title{
Galactic warp from the kinematics of OB stars
}

\author{
Rizky Maulana Nurhidayat ${ }^{1, *}$, Mochamad Ikbal Arifyanto ${ }^{2, * *}$, and Lucky Puspitarini ${ }^{2}$ \\ ${ }^{1}$ Astronomy Study Program, Faculty of Mathematics and Natural Sciences, Institut Teknologi Bandung, \\ Indonesia \\ ${ }^{2}$ Astronomy Research Division, Faculty of Mathematics and Natural Sciences, Institut Teknologi Ban- \\ dung, Indonesia
}

\begin{abstract}
The warp in Milky Way seen through the young stellar disk shows the complex structures. Its spatial distribution reveals the asymmetry in the vertical distance to the galactic midplane, whereas the kinematics shows the oscillating motion. We analyze the warp using about 25,000 OB stars from Gaia Data Release 2, with Galactocentric azimuth $(\phi)$ range from $135^{\circ}$ to $225^{\circ}$. We detect the warp from two distinctive regions. First region (warp down) lies on $100^{\circ}<l<150^{\circ}$ and second regions (warp up) lies on $150^{\circ}<l<220^{\circ}$. The vertical position towards the Galactic midplane $(Z)$ from all samples shows the line-of-nodes of the warp is on $\phi=174^{\circ} \pm 2^{\circ}$. The maximum deviation in warp down region reachs $0.1 \mathrm{kpc}$ and $0.2 \mathrm{kpc}$ in warp up region. The vertical velocity $\left(V_{Z}\right)$ from both regions are dominated by negative $V_{Z}$. From the kinematics distribution, it can be concluded that there is an oscillatilng motion. Considering with the previous study, the oscillating motion was caused by the gravitational interaction with the Galaxy satellites. From this, we can constrain the origin of the warp is developing from the tidal interaction between Milky Way and its satellites.
\end{abstract}

\section{Introduction}

A common feature in a spiral galaxy is a warped disk. Analyzing edge-on spiral galaxies from Sloan Digital Sky Survey and NASA Extragalactic Database, there are 236 from 325 galaxies that develop warp, with 165 galaxies have S-shaped warp and 71 galaxies have Ushaped warp [1]. Warp also develops in the nearest galaxy, M31. The integral-sign warp starts at $R \simeq 30 \mathrm{kpc}$ from the galaxy center of M31 with a deviation of more than $3 \mathrm{kpc}$ to the galaxy midplane [2].

The study about warp in Milky Way comes from the analysis of the spatial distribution of HI gas [3] and the young-old stellar population [4]. They found that the Milky Way develops aligned warp both in the HI gas and stellar distribution. The second quadrant of Galactocentric azimuth $(\phi)$ is dominated by gas and stars located above the Galaxy midplane. The opposite features are shown in the third quadrant. The deviation of the warp is dependent on the tracers' age [4]. The warp from the young stellar population (OB stars) deviates of 0.23 $\mathrm{kpc}$ and $0.19 \mathrm{kpc}$ on the warp up and warp down region, respectively. The warp developing in the old stellar population (RGB stars) reaches up to $0.97 \mathrm{kpc}$ at the warp up region and

\footnotetext{
*e-mail: rizman.nana@students.itb.ac.id

**e-mail: ikbal@as.itb.ac.id
} 
$1.22 \mathrm{kpc}$ at the warp down region [4]. From another young stellar tracer like Cepheid stars, the warp starting develops at $9.26 \mathrm{kpc}$ with line-of-nodes (LoN) of $17.4^{\circ}$ [5]. The kinematics distribution of Upper Main Sequence (UMS) that is called OB stars in this paper and Red Giant Branch (RGB) star in Gaia Data Release 2 (DR2) shows the increasing vertical velocity in the anti-center direction [6].

The origin of the warp is still debatable. It can be a product of a misaligned inner and outer halos in the dynamics of the galaxy [7], the misaligned of the angular momentum axis with the disk spin axis of the late infall [8], tidal interaction between galaxy satellites and its host $[9,10]$ and the interaction between galaxy magnetic fields with the intergalactic magnetic fields [11]. We collect the phase-space motion of OB stars from Gaia DR2 [12] cross-matched with LAMOST DR4 [13], RAVE DR5 [14], GALAH DR2 [15], 2nd. Catalog of Radial Velocities with Astrometric Data (hereafter CRVAD) [16], and Pulkovo Radial Velocities for 35493 HIP Stars (hereafter PRV) [17] to analyze the Milky Way warp and constrain its origin.

\section{Data and Methods}

To select OB stars from Gaia DR2, we apply initial criteria as mentioned in [18], shown in equation (1) and (2).

$$
\begin{gathered}
M_{G}=G+5 \log \varpi-A_{G}-10<2 \\
\left(G_{B P}-G_{R P}\right)_{0}=\left(G_{B P}-G_{R P}\right)-E\left(G_{B P}-G_{R P}\right)<0,
\end{gathered}
$$

where $\varpi$ is the parallax of the stars in mas, $G, G_{B P}, G_{R P}, E\left(G_{B P}-G_{R P}\right)$, and $A_{G}$ are photometric in G-band $(330-1050 \mathrm{~nm}), G_{B P}(330-680 \mathrm{~nm}), G_{R P}(630-1050 \mathrm{~nm})$, color excess, and extinction were provided by Gaia DR2, respectively.

The next step is excluding the contamination of giants or red clump stars in OB star candidates we get from equation (1) and (2), with applying equation (3) and (4).

$$
\begin{gathered}
J-H<0.14\left(G-K_{S}\right)+0.02 \\
J-K_{S}<0.23\left(G-K_{S}\right),
\end{gathered}
$$

where $J, H$, and $K_{S}$ are the photometric in $J H K_{S}$-bands provided by 2 MASS. The distance for each OB stars is calculated with equation (5) that is valid for $\varpi>0$ and $\varpi_{e r r} / \varpi<0.2$ as mentioned in [19], where $\varpi_{e r r}$ is the uncertainty of parallax in mas.

$$
D=\frac{1}{\varpi}
$$

where $D$ is in kpc. To ensure our OB samples, we exclude the stars which have vertical distance from galactic midplane $(|Z|)$ of more than $2 \mathrm{kpc}$. Finally, we find the radial velocity from OB stars that not provided in Gaia DR2 by cross-matching with LAMOST DR4, RAVE DR5, GALAH DR2, CRVAD, and PRV. We get phase-space coordinates of 24,958 OB stars.

We use a galaxy model with the distance of Sun-Galactic Center $X_{\odot}=-8.34 \mathrm{kpc}[20]$ and the distance of Sun-Galactic midplane $Z_{\odot}=0.027 \mathrm{kpc}$ [21]. The coordinate transformation, from Galactic coordinates to cartesian Galactocentric coordinates, is shown in equation (6), (7), and (8).

$$
\begin{gathered}
X=D \cos b \cos l-X_{\odot} \\
Y=D \cos b \sin l \\
Z=D \sin b+Z_{\odot},
\end{gathered}
$$


where $l$ and $b$ are the Galactic coordinates of each star, $X, Y$, and $Z$ is the position concerning the Galactic Center (GC), adopting the right-handed system. To transform in cylindrical Galactocentric, we apply equation (9) and (10).

$$
\begin{gathered}
R=\sqrt{X^{2}+Y^{2}} \\
\phi=\arcsin \left(\frac{D \sin l}{R}\right) .
\end{gathered}
$$

We analyze the distribution of Milky Way warp from the XY projection with the colorcoded of $Z$. Then, we find LoN of the warp from our samples with determining the $Z$ as a function of $\phi$ using the transformation in equation (10), with $R$ is the Galactocentric radius and $\phi$ that starting from positive of $X$-axis increases in the opposite direction of the galactic rotation. We bin the data for every 500 stars, with no overlapping. To analyze the kinematics distribution of the warp from our samples, we measure the vertical velocity $\left(V_{Z}\right)$ as a function of $\phi$, with no overlapping 150 stars for each bin.

\section{Results and Analysis}

The spatial distribution of our OB stars in the XY projection is shown in Figure 1. Our OB stars is distributed in about $135^{\circ}<\phi<225^{\circ}$. Figure 1 shows the complex distribution, in line with the previous study [4]. The color-coded in Figure 1 shows the mean $\mathrm{Z}$ for each bin with a size of $0.1 \mathrm{kpc} \times 0.1 \mathrm{kpc}$. The solid gray line indicates the Galactocentric radius of 8 , 10,12 , and $14 \mathrm{kpc}$ with a red dot is the position of the Sun. We detect the warp developed in the blue and red regions. The blue region called warp up region, lies on Galactic longitude $100^{\circ}<l<150^{\circ}$, and the red region called warp down region, lies on $150^{\circ}<l<220^{\circ}$.

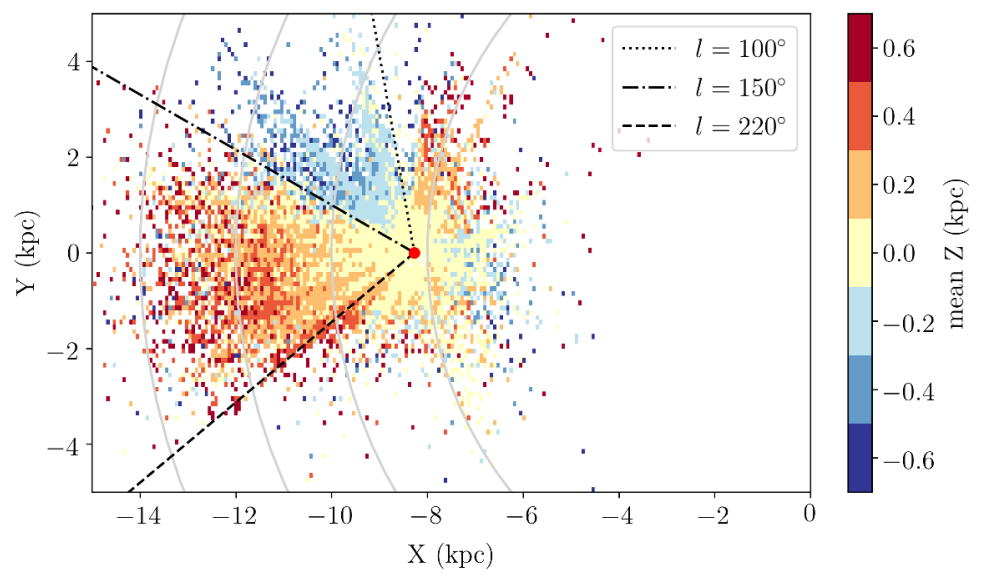

Figure 1. XY projection of OB stars in about $135^{\circ}<\phi<225^{\circ}$. Four gray solid lines are $R=8,10,12$, and $14 \mathrm{kpc}$ with mean $\mathrm{Z}$ as a color-code. Sun is presented by a red dot. Warp down and warp up region are selected in $100^{\circ}<l<150^{\circ}$ and $150^{\circ}<l<220^{\circ}$, respectively.

In analyzing LoN of the warp from our OB stars, we measure the mean of $\mathrm{Z}$ as a function of $\phi$, shown in Figure 2. The error bar for each data point comes from the standard deviation in each bin. We can determine LoN lies at $\phi=174^{\circ} \pm 2^{\circ}$. The uncertainty of LoN is calculated by the mean $\phi$ of two nearest data point to $Z=0 \mathrm{kpc}$. From this result, the Sun is not located 
on LoN, in line with the previous study [22]. The maximum deviation below the galactic midplane reaches about $0.1 \mathrm{kpc}$, at $\phi=170^{\circ}$ and the deviation above the galactic midplane reaches $0.2 \mathrm{kpc}$ at $\phi=190^{\circ}$. The asymmetry of Milky Way warp also found in the previous study [4, 23], with different representations. If we assume the mean $\mathrm{Z}$ in Figure 2 can be approximated with sinusoidal function, several data points in $177^{\circ}<\phi<182^{\circ}$ will be out of the function. We guess that it is caused by the presence of the North Near (NN) overdensity [24]. We get more stars in NN lies on $177^{\circ}<\phi<182^{\circ}$ and one data point at $\phi=187^{\circ}$, but less stars in other region of NN.

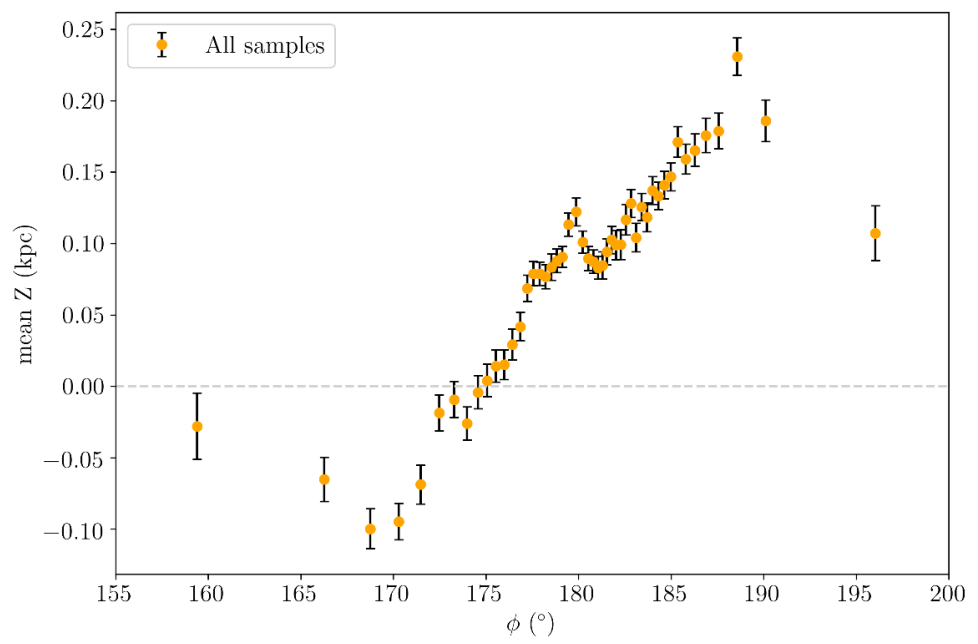

Figure 2. Mean $\mathrm{Z}$ as a function of $\phi$ for all samples. Each data point is consisted of 500 stars, with no overlapping. The gray dashed line is the position of galactic midplane.

The kinematics distribution of the warp is shown in Figure 3. We analyze two regions found in Figure 1, warp down and warp up region are represented by red and blue data point, respectively. The binning method is similar to the previous binning applied in Figure 2, with the number of stars for each bin is 150 . The kinematics distribution of the warp down region, $(Z<0)$, is dominated by the negative $V_{Z}$. We get a similar distribution in the warp up region, that has $Z>0$. It can be expected that both of the regions are not static. It is like a part of oscillation. Because of this oscillation, the LoN of Milky Way warp will be shifted. Comparing with the previous work [25], we guess that the oscillation we found is just a small part of vertical damped oscillation in [25]. The damped oscillation is expected to come from the interaction between dwarf Sagittarius with Milky Way. So, we constrain the origin of the warp we detected is coming from the dwarf Sagittarius interaction in orbiting Milky Way.

\section{Conclusion}

The Milky Way warp from OB stars reveals a complex spatial distribution. In the kinematics distribution, we can conclude that the warp is not a static feature. The warp down and warp up region we found are dominated by negative $V_{Z}$. It shows the vertical oscillation motion of the stars, just in a small region. Considering the study of the perturbed Milky Way disk, our result is in line with the previous study [25]. From these oscillations, the origin of the Milky Way warp can be constrained. It could be a product of the interaction of the galactic satellites 


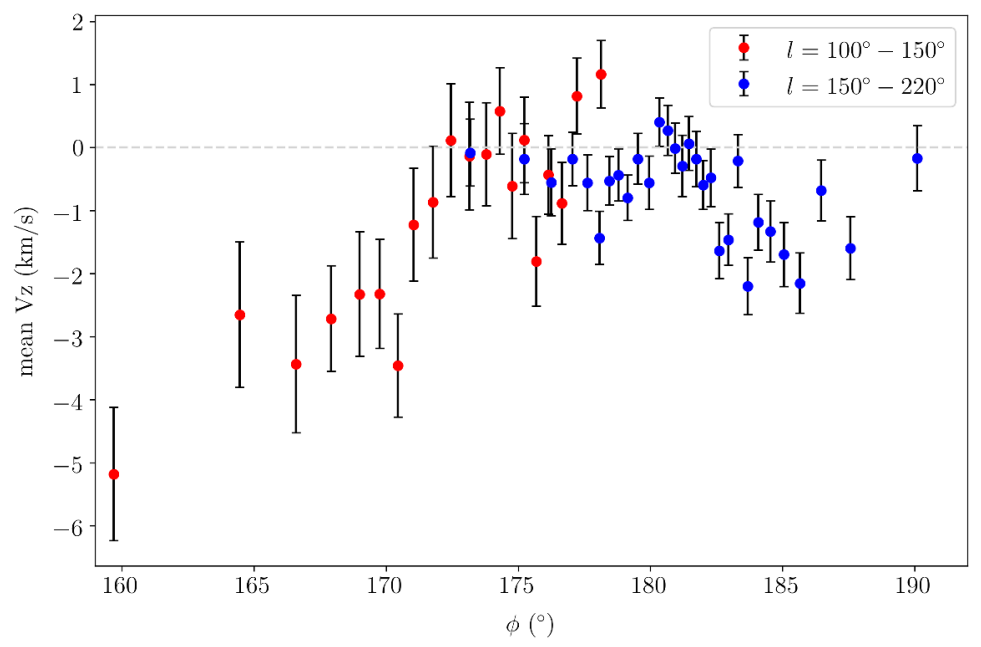

Figure 3. Mean $V_{Z}$ as a function of $\phi$ for warp down and warp up region. Warp down and warp up region are presented by red dots and blue dots, respectively. Each data point is consisted of 150 stars, with no overlapping.

with Milky Way. In this case, dwarf Sagittarius that has a polar orbit is expected as a culprit. A more detailed study is needed to ensure the dynamics of the Milky Way warp in the past by analyzing the spatial and kinematics from the different stellar populations and building a model of the warp.

\section{Acknowledgement}

The authors gratefully acknowledge a research grant from the Program of Research, Community Service, and Innovation of the Institut Teknologi Bandung (P3MI-ITB). This work has made use of data from the European Space Agency (ESA) mission Gaia (https://www. cosmos.esa.int/gaia), Guoshoujing Telescope (the Large Sky Area Multi-Object Fiber Spectroscopic Telescope LAMOST) survey, Radial Velocity Experiment (RAVE) survey, The Galactic Archaeology with HERMES (GALAH) survey, VizieR Online Data Catalog: 2nd Cat. of Radial Velocities with Astrometric Data, and VizieR Online Data Catalog: Pulkovo radial velocities for 35493 HIP stars.

\section{References}

[1] H.B. Ann, J-C. Park, New Astron. 11, 293 (2006)

[2] K. Newton, D.T. Emerson, MNRAS 181, 573 (1977)

[3] E.S. Levine, L. Blitz, C. Heiles, ApJ 643, 881 (2006)

[4] M. Romero-Gómez, C. Mateu, L. Aguilar, F. Figueras, A. Castro-Ginard, A\&A 627, A150 (2019)

[5] X. Chen, S. Wang, L. Deng, R. de Grijs, C. Liu, H. Tian, Nature Astronomy 3, 320 (2019)

[6] E. Poggio, R. Drimmel, M.G. Lattanzi, R.L. Smart, A. Spagna, et al., MNRAS 481, L21 (2018)

[7] J. Dubinsky, D. Chakrabarty, ApJ 703, 2068 (2009) 
[8] J.A. Sellwood, TD Oswalt \& G. Gilmore 923, (2013)

[9] F.A. Gómez, I. Minchev, B.W. O’Shea, T.C. Beers, J.S. Bullock, C.W. Purcell, MNRAS 429, 159 (2012)

[10] J.H. Kim, S. Peirani, S. Kim, H.B. Ann, S. An, S. Yoon, ApJ 789, 90 (2014)

[11] E. Battaner, J. Jimenez-Vicente, arXiv preprint astro-ph/9802020 (1998)

[12] Gaia Collaboration, T. Prusti, J.H.J. De Bruijne, A.G.A. Brown, A. Vallenari, et al., A\&A 595, A1 (2018a)

[13] A.L. Luo, Y.H. Zhao, G. Zhao, et al., Vizier Online Data Catalog (2019)

[14] A. Kunder, G. Kordopatis, M. Steinmetz, T. Zwitter, et al., AJ 153, 75 (2016)

[15] S. Buder, M. Asplund, L. Duong, J. Kos, et al., MNRAS 478, 4513 (2018)

[16] N.V. Kharchenko, R.D. Scholz, A.E. Piskunov, S. Roeser, E. Schilbach, Vizier Online Data Catalog (2007)

[17] G.A. Gontcharov, Vizier Online Data Catalog (2006)

[18] Gaia Collaboration, D. Katz, T. Antoja, M. Romero-Gómez, et al., A\&A 616, A11 (2018b)

[19] C.A.L. Bailer-Jones, PASP 127, 994 (2015)

[20] M.J. Reid, K.M. Menten, A. Brunthaler, et al., ApJ 783, 130 (2014)

[21] B. Chen, C. Stoughton, J. A. Smith, et al., ApJ 553, 184 (2001)

[22] C. Li, G. Zhao, Y. Jia, S. Liao, C. Yang, Q. Wang, ApJ 871, 208 (2019)

[23] I. Yusifov, The Magnetized Interstellar Medium: Proc. Conference held in Antalya, Turkey (eds Uyaniker, B., Reich, W. \& Wielebinski, R.) 165-169 (Copernicus GmbH, 2004).

[24] Y. Xu, H.J. Newberg, J.L. Carlin, C. Liu, L. Deng, J. Li, R. Schönrich, B. Yanny, ApJ 801, 105 (2015)

[25] T. Antoja, A. Helmi, M. Romero-Gómez, D. Katz, C. Babusiaux, R. Drimmel, D.W. Evans, F. Figueras, E. Poggio, C. Reylé, A.C. Robin, G. Seabroke, C. Soubiran, Nature 561, 360 (2018) 